\title{
A Practical, Clinical User-Friendly Format for Breast Ultrasound Report
}

\author{
(1) Sadaf Alipour ${ }^{1,2}$, (1) Bita Eslami ${ }^{1}$, (10) Mahboubeh Abedii ${ }^{1,3}$, (10) Nasrin Ahmadinejad ${ }^{4,5}$, (1) Ali Arabkheradmand ${ }^{6}$, \\ (D) Arvin Aryan ${ }^{4}$, (D) Khadijeh Bakhtavar ${ }^{7}$, (D) Leila Bayani ${ }^{1,3}$, (D) Ahmad Elahi ${ }^{1,8}$, (D) Masoumeh Gity ${ }^{1,4}$, (D) Maryam Rahmani ${ }^{4}$, \\ (D) Nahid Sedighi ${ }^{4}$, (D) Adel Yazdankhahkenari ${ }^{9}$, (D) Ramesh Omranipour ${ }^{1,10}$ \\ ${ }^{1}$ Breast Disease Research Center, Tehran University of Medical Sciences, Tehran, Iran \\ ${ }^{2}$ Department of Surgery, Arash Women's Hospital, Tehran University of Medical Sciences, Tehran, Iran \\ ${ }^{3}$ Department of Radiology, Arash Women's Hospital, Tehran University of Medical Sciences, Tehran, Iran \\ ${ }^{4}$ Department of Radiology, Advanced Diagnostic and Interventional Radiology Research Center (ADIR), Tehran University of Medical Sciences \\ (TUMS), Tehran, Iran \\ ${ }^{5}$ Medical Imaging Center, Cancer Research Institute, Imam Khomeini Hospital, Tehran, Iran \\ ${ }^{6}$ Department of Surgery, Cancer Institute, Tehran University of Medical Sciences, Tehran, Iran \\ ${ }^{7}$ Department of Radiology, Sina Hospital, Tehran University of Medical Sciences, Tehran, Iran \\ ${ }^{8}$ Division of Breast Surgical Oncology, Department of Surgery, Alborz University of Medical Sciences, Karaj, Iran \\ ${ }^{9}$ Trauma and Surgery Research Center, Sina Hospital, Tehran University of Medical Sciences, Tehran, Iran \\ ${ }^{10}$ Department of Surgical Oncology, Cancer Institute, Tehran University of Medical Sciences, Tehran, Iran
}

\section{ABSTRACT}

Objective: Breast ultrasound (BUS) is often performed as an adjunct to mammography in breast cancer screening or for evaluating breast lesions. Our aim was to design a practical and user-friendly format for BUS that could include the details of the Breast Imaging Reporting and Data System.

Materials and Methods: As a team of radiologists and surgeons trained in the management of breast diseases, we gathered and carried out the project in four phases-literature search and collection of present report formats, summarizing key points and preparing the first draft, seeking expert opinion and preparing the final format, and pilot testing-followed by a survey was answered by the research team's radiologists and surgeons.

Results: It produced a list of items to be stated in the BUS report, the final BUS report format, and the pilot format guide. Then, the radiologists used the format in three active ultrasound units in university-affiliated centers, and reports were referred to the surgeons. At the end of the project, the survey showed a high degree of ease of use, clarity, conciseness, comprehensiveness, and well-classified structure of the report format; but radiologists believed that the new organization took more time.

Conclusion: We propose our design as a user-friendly and practical format for BUS reports. It should be used for a longer time and by various ultrasound centers in order to ascertain its benefits.

Keywords: Breast, ultrasonography, breast diseases

Cite this article as: Alipour S, Eslami B, Abedi M, Ahmadinejad N, Arabkheradmand A, Aryan A, Bakhtavar K, Bayani L, Elahi A, Gity M, Rahmani M, Sedighi N, Yazdankhahkenari A, Omranipour R. A Practical, Clinical User-Friendly Format for Breast Ultrasound Report.

Eur J Breast Health 2021; 17(2): 165-172

\section{Key Points}

- Breast ultrasound is one of the most frequently used modality in breast screening.

- $\quad$ BUS can detect and define lesions and assist both in diagnosis and treatment planning of breast disorders.

- An applied format for BUS report that could be user-friendly for breast care practitioners was designed and tested. 


\section{Introduction}

Breast cancer is the most prevalent female cancer and the first cause of death from cancer in women worldwide $(1,2)$. Breast cancer screening is achieved by clinical breast examination (CBE) and mammography, but under numerous circumstances, breast ultrasound (BUS) is used as a complementary modality (3). Breast complaints are also among the most common causes for women to attend surgery and gynecology clinics (4). In addition to breast examination, many cases need to be further examined by imaging, and BUS is one of the most frequently used modalities in this regard. Also, in many referral centers, breast surgeons regularly use ultrasound imaging as an adjunct to clinical examinations.

BUS can detect and define lesions and assist both in diagnosis and treatment planning of breast disorders, especially in discrimination of solid and cystic masses, which is beyond the diagnostic field of mammography, and in detection of hidden masses in dense breast mammogram. However, in many situations, lesions detected by BUS undergo serial ultrasound to follow the probable changes of that specific lesion [usually Breast Imaging Reporting and Data System (BIRADS) III Category: a probably benign lesions], in order to discriminate benign and suspicious ones.

How to define findings in BUS and which features to note in the report have been described in the ultrasound lexicon and the Breast Imaging Reporting and Data System (5).

A precise BUS report denoting all details is certainly helpful, but two key problems arise. First, which of these details would help the incharge physicians in medical decision-making and would affect the management plan? Second, how should the arrangement of the report be in order to make it practical and easy to use? In other words, the user of a BUS report is the physician that is managing the breast disorder, who is usually a breast or general surgeon, a gynecologist, or perhaps a family practitioner general physician.

The format of the report, the arrangement of the details, and the number of significant or nonsignificant details affect the practicality and usefulness of the report. A wisely organized report, with the details applied in an orderly manner, would save the clinicians' time and help them figure out the clinically significant points aptly.

In addition, all BUS must be compared with the previous ones in order to identify changes in previous lesions or new findings. At present, various ultrasound units use different formats, although many observe the key problems of the BIRADS system and lexicon. These various styles may make the comparison between ultrasound reports very time-consuming and sometimes ambiguous. A comparison would be simpler if all BUS reports were arranged systematically and uniformly and especially if they were all arranged in the same form.

As a team of radiologists and clinicians whose main field of interest is the management of breast diseases, we have carried out a study to design the applied format for BUS report, which would yield the above advantages.

\section{Materials and Methods}

This project was supported by the Vice-Chancellor in Research assessed and ethically approved by its Ethics Committee (ethics code: IR.TUMS.VCR.REC.1 397.846).

We formed a team including radiologists and surgeons who are expert in the management of breast diseases. All radiologists were dedicated to gynecological imaging or breast imaging and have practiced in the radiology departments of Tehran University of Medical Sciences (Tehran), which ranks first in education and research among medical universities of the country. In Iran, as in many other countries, patients undergo ultrasound in different centers on the basis of which they can book an appointment. In addition, radiologists generally mention all the lesions they detect in the BUS in their reports, although unfortunately most clinicians do not mention an individual target lesion to be assessed when they are requesting the BUS. In our country, surgeons are responsible for the clinical management of breast diseases, and surgical oncologists and breast surgical oncologists are trained and entitled for subspecialized practice over the subject. All the surgeons of our research team were surgical oncologists or breast surgical oncologists and practiced as full-time or part-time professors at our university. We performed the study in the following four phases.

\section{Phase 1: Literature search and collection of present report formats}

This phase consisted of two stages that were accomplished by one of the surgeons and a research expert. In the first stage, an extended search of the English literature from 1990 to the present time was performed for similar works and different viewpoints about the ultrasound BIRADS lexicon. The rationale for beginning the search from the 1990s was because the first version of BIRADS was issued in 1993 by the American College of Radiology (ACR) (6). The keywords consisted of BUS report, BIRADS ultrasound, BUS interpretation, breast imaging report, and breast mass radiologist assessment. All articles containing relevant data or viewpoints were gathered. Also, chapters or paragraphs about the subject in referral radiology or breast books were investigated in this stage.

In the second stage, we collected BUS reports from high-volume and low-volume radiology centers in Tehran, the capital of Iran, and from several centers in large or small cities around the country. In order to provide a basis for detection of defects of the reports and compare them, we outlined them grossly as four types of formats, which are summarized in Figure 1.

\section{Phase 2: Summarizing key points and preparing the first draft}

Two of the surgeons completed this phase in three steps. In the first step, the key points in the BUS report were extracted from the ultrasound BIRADS lexicon and the few related returned articles in our search. In the second step, all collected reports were reviewed and evaluated regarding precision, clarity, and ease to use of the arrangement, as well as ambiguous, vague, or complicated definitions or organization of the items in the second step.

In the third step, the items to be defined in a BUS report were designated according to the BIRADS lexicon; and the most appropriate order for the reporting of those items was argued, in an effort to describe an order which could provide the highest clarity. Several designs were prepared as drafts to be discussed.

\section{Phase 3: Seeking expert opinion and preparing the final format}

The third phase consisted of an expert panel, attended by two breast surgeons, three surgical oncologists, eight breast and gynecologic radiologists, and a research expert and then several virtual meetings 


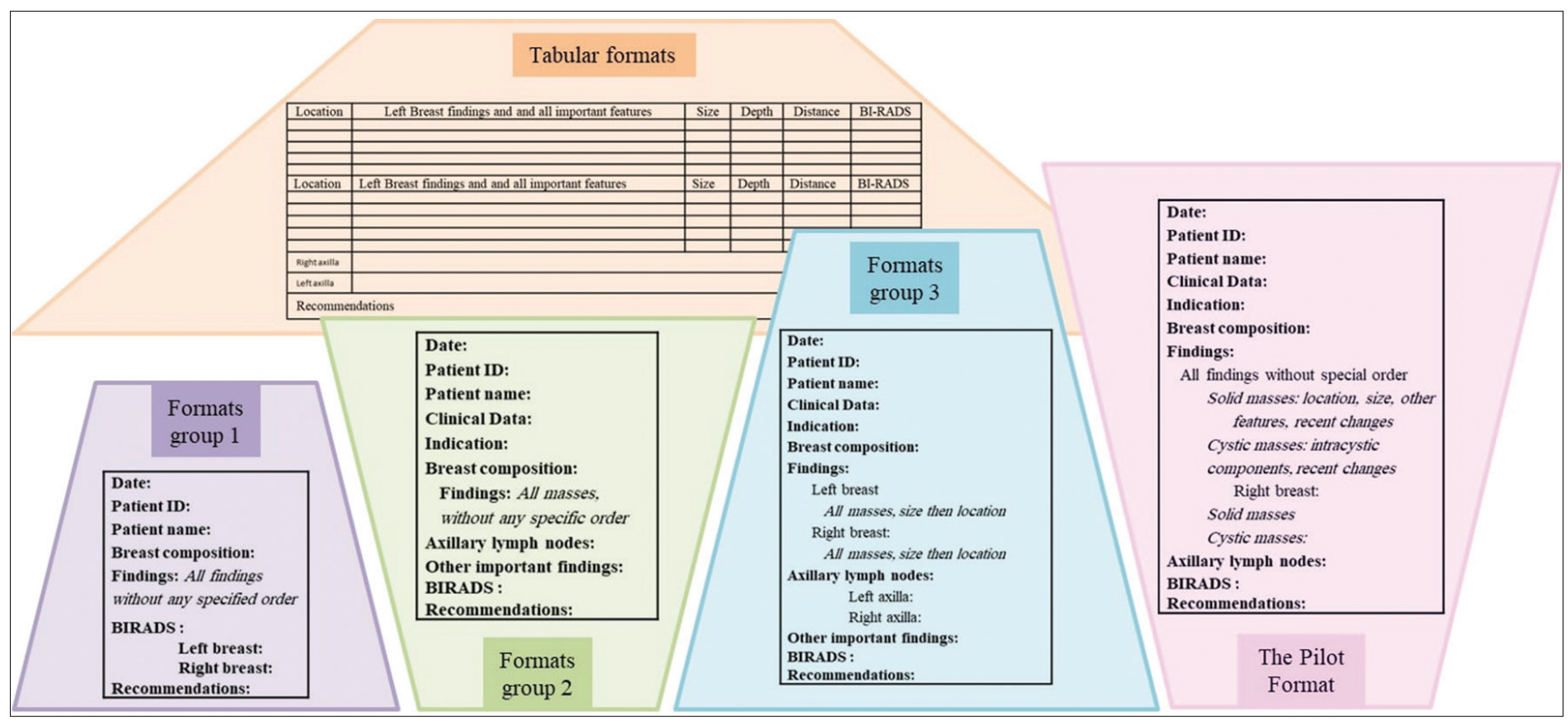

Figure 1. General gross classification of the frameworks of existing breast ultrasound report and comparison with the approved pilot format (all frameworks are shortened to fit in the figure)

ID: Identification; BIRADS: Breast Imaging Reporting and Data System

in the era of coronavirus disease-2019 (COVID-19) through a virtual group including all the named experts as members. The drafted designs for BUS report were introduced and debated during the meetings. One design was designated as most user-friendly, and further modifications were proposed. After several revisions, a final framework was defined and approved as a BUS report pilot format.

\section{Phase 4: Pilot testing}

The last phase consisted of pilot testing of the approved format. This was supposed to be uniformly held in ultrasound units of university hospitals for 4 months, so that a comparison of two subsequent results could take place in some cases that underwent two BUS in a 3-month interval. Due to COVID-19 conditions and the delay in many schedules including holding of most screening programs, the number of monthly BUS dropped largely; however, three major units remained active, although with a small number of patients. These were the centers where the radiologists and surgeons of the research team were practicing. Therefore, the pilot was held in these three units for around 5.5 months. After this time, a survey was carried out to assess the format from the point of view of the research team's radiologists and surgeons.

\section{Results}

The first product of the panel was the list of items to be stated in the BUS report based on the ultrasound BIRADS lexicon, as demonstrated in Table 1.

The second product was the final BUS report format, which was proposed as a straightforward, user-friendly framework for reporting BUS. Since the format could only demonstrate the basic structure for writing the report, a guideline (the pilot format guide) was also written to explain how and where to describe the items in the framework. Table 2 illustrates the BUS report pilot format, and Table 3 shows the pilot format guide.
Following the establishment of the program in the three units, radiologists' reports were printed according to the proposed format, and the patients brought them to their surgeons according to their schedules.

A brief survey was designed to investigate the impression of the specialists about the new format, and the responses were rated on a 5-point Likert scale: strongly disagree, disagree, undecided, agree, and strongly agree. The survey contained 11 questions, as seen in Table 4. After 5.5 months project execution, all surgeons and radiologists filled the survey. The results of the survey for each group and for all experts are demonstrated in Figure 1 and Figure 2. The average number of BUS performed by each radiologist and seen by each surgeon per month is approximately 200 cases in non-COVID-19 conditions.

\section{Discussion and Conclusion}

We performed a study to design and test an applied format for BUS report that could be user-friendly for breast care practitioners. After gathering the existing formats and assessing them, we defined a framework and its user guide through several panels and tested it in three high-volume BUS units, with favorable outcomes.

The sensitivity of mammography in detecting suspicious lesions is variable and is overall lower in dense breast tissue $(3,7,8)$. Adding BUS to mammography increases the sensitivity for detection of breast cancer in women with high mammographic breast tissue density (9). Berg et al. (10) performed a multicenter study involving 2809 women at high risk for breast cancer to find out if the inclusion of BUS to mammography may have an effect in the diagnostic yield of the latter during breast cancer screening. They showed a diagnostic yield of 7.6 versus 11.8 per 1,000 women screened for mammography alone and the combination of the two modalities, respectively (2). Gharekhanloo et al. (11) also confirmed the additional sensitivity provided by adding BUS to mammography for the detection of breast cancer in their study on 300 cases. The additional advantage of BUS in mammographic 
Table 1. Items to be stated in the BUS report as approved in the expert panel

\section{General items}

History

Family history

Indication

Breast composition

\section{Findings}

Mass

Tissue distortion

Retraction

Calcification

Lymphadenopathies

Skin changes

Nipple changes

Postoperative findings

Descriptions for any breast finding

Side

Location

Distance (mm)

Depth (mm)

Comparison

\section{Descriptions for masses}

Size $(\mathrm{mm})$

Type

Echopattern

Shape

Lobulations

Margins

Orientation

Posterior features

Vascularity

Elasticity

Intracystic details

Postoperative findings

Significant recent change in findings

Correlation with mammographic, MRI, or clinical findings

BIRADS

Recommendations of the sonographer

BUS: Breast ultrasound; BIRADS: Breast Imaging Reporting and Data System; MRI: Magnetic resonance imaging

breast cancer screening in women at high risk of breast cancer has been maintained by the American College of Radiology Imaging Network via a multicenter trial (12).

In addition to screening purposes, BUS assists in the evaluation of breast symptoms and signs, including lumps or nipple discharge.
Previous breast medical and surgical history or previous biopsy results

Of breast cancer

For performing the BUS

Homogenous background echotexture-fat/homogenous background echotexture-fibroglandular/ heterogenous background echotexture

Described as below

Described as below

Described as below

Described as below

Location (axillary, in breast), significance, cortical thickness, hilum changes, extracapsular invasion, matted nodes

Edema, thickness, retraction

Retraction

Left/right

On a clock face

From the nipple

From the skin

Comparison with previous ultrasound examination

The largest dimension or the three dimensions

Cystic, solid

Anechoic, hypoechoic, isoechoic, hyperechoic, heteroechoic

Round, oval, irregular

Microlobulations, macrolobulations; number

Circumscribed, indistinct, angular, spiculated

Horizontal, vertical

None, enhancement, shadowing, combined pattern

Absent, internal, vessels in rim

Soft, intermediate, hard

Septations, masses

regarding their shape, size, or other features; a part of the follow-up is performed by serial BUS. Hence, overall, BUS plays a significant role in the approach to the breast.

In 2003, the ACR released a BIRADS lexicon for ultrasound that intended to standardize BUS reports and simplify comparisons with previous imaging (5). This has yielded a kind of international shared 


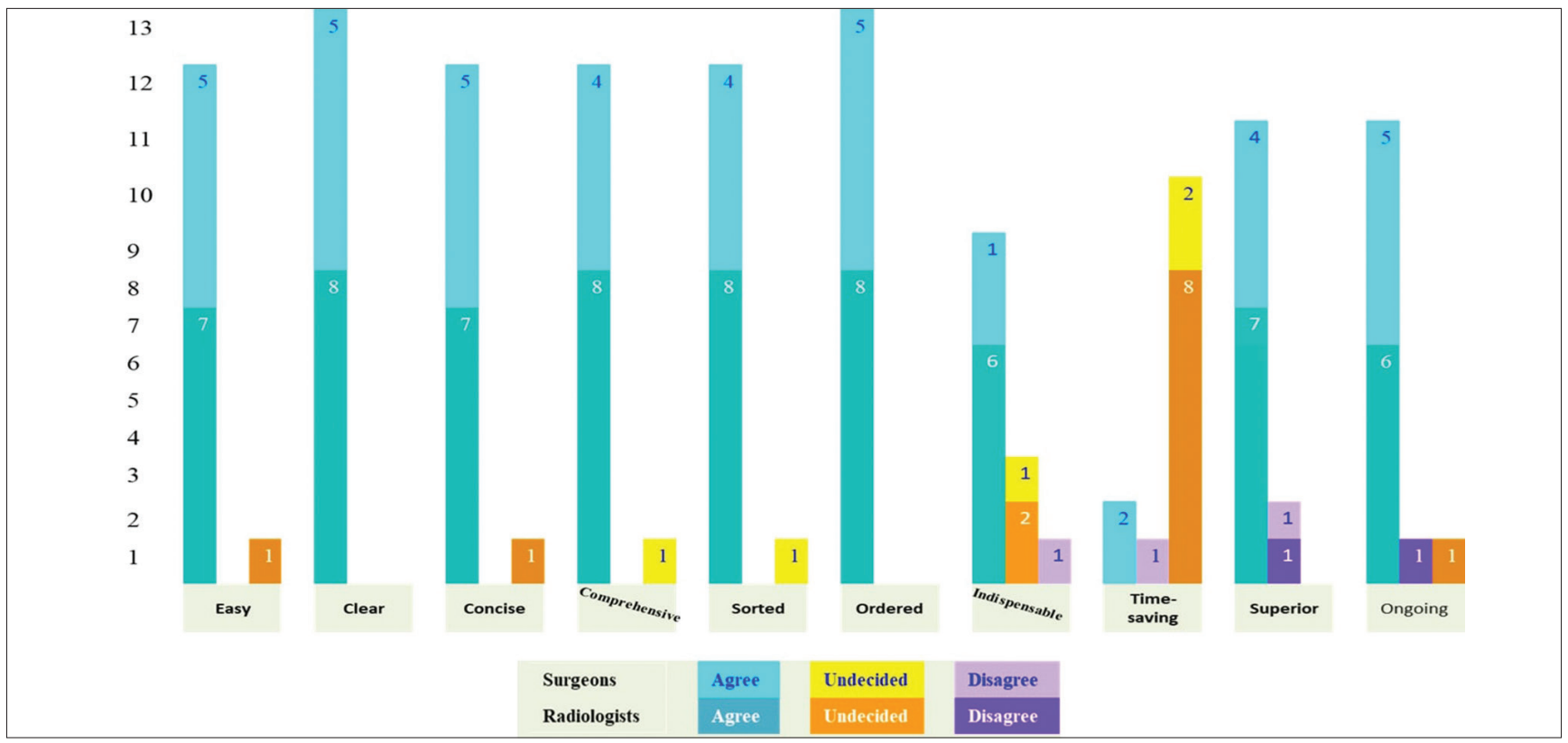

Figure 2. Results of survey among radiologists and surgeons

Table 2. Framework of approved pilot format
- Date:
- Patient ID:
- Patient name:
- Clinical data:
- Indication:
- Breast composition:
- Findings:

\section{* Left breast \\ $>$ Solid masses}

-

$>$ Cystic masses

-

\& Right breast:

$>$ Solid masses

-

> Cystic masses:

-

- Axillary lymph nodes:

o Left axilla:

-

o Right axilla:

-

- Other important findings:

O

- BIRADS:

o Left breast:

o Right breast:

- Recommendations:

ID: Identification; BIRADS: Breast Imaging Reporting and Data System language between radiologists. Abdullah et al. (13) evaluated the concordance of definitions of five sonographists about ultrasound characteristics of 267 benign and malignant breast lumps based on the BIRADS lexicon for BUS. They detected an overall "good" level of agreement but a fair one for evaluating lesion borders. The concordance of their description was lower for smaller lumps as well as malignant cases. Their overall conclusion was in favor of a good interobserver agreement. This was confirmed by the studies of Lazarus et al. (14) and Costantini et al. (15) on 91 and 178 breast lesions, respectively.

While BUS is performed by radiologists, the clinician has to decide on the suitable approach to a breast lesion based on the findings of breast exam and breast imaging. Consequently, the BIRADS system and the lexicon also aim to ease the communication between the sonographer and the clinician. As a creditable product should be produced by the cooperation of stakeholders with diverse viewpoints from different aspects, several medical organizations have cooperated in the production of the BIRADS lexicon, including associations of surgeons, who could be seen as the end-users of the lexicon product, or the BUS report (16). Items that should be mentioned in a report, descriptors for every item, and the gross order of the report are explained in the BIRADS lexicon for BUS. However, the order of the details, the scope and number of details, and the visual method for emphasizing on more important findings can also be outlined, giving rise to user-friendly reports that could easily be compared. This is what our team aimed for, by delineating an orderly structure for the BUS report, where details appear in accord with the BIRADS lexicon, and the usual classifications of breast lesions. In our proposed format, sorting the lesions by type allows users to selectively pick up the parts they are concerned about or first pay attention to components that are more important to them. By writing the clockface location of each lesion first, the users localize the lesion in their mind and match it with the CBE or other imaging modalities. The size of each lesion immediately follows, because size change is almost the most important alteration that can affect the significance of a finding. Then, the other location coordinates including depth from the skin and distance from the nipple depicted as near zone, mid zone, and far zone are described 
Table 3. Guide for approved pilot format

- Date:

- Patient ID:

- Patient name:

- Clinical data (age, history, family history)

- Indication (cause for requesting ultrasound)

- Tissue composition (according to ACR format: homogenous background echotexture-fat/homogenous background echotexturefibroglandular/heterogenous background echotexture)

Findings:

For suspicious lesions: please write in BOLD + mention ZONE (near zone, mid zone, far zone) and DEPTH (anterior zone, mid zone, posterior zone) + write the BIRADS of that specific lesion

For new lesions or lesions with recent changes, please write in BOLD

For lesions in location of clinician interest, please write in BOLD

- For any suspicious finding in the breast other than masses, like tissue distortion or retraction, please write it next to the mass or in the relevant location among masses

If typical, please write the probable diagnosis of the mass (probably fibroadenoma, fat necrosis, hamartoma, intramammary lymph node...)

For any significant finding, if correlated with mammographic, MRI, or clinical findings, please mention it, with BIRADS

Please follow this order:

* Left breast:

$>$ Solid masses

- In order of clock hours, first retroareolar, then 1 to 12

(iti In each line, please first write the location (.... O'clock) and the size, then if needed the zone (NZ, MZ, FZ) and the depth (....mm from skin), then the features of the mass as needed (irregular margin, orientation, posterior features, vascularity, elasticity, ...)

\section{$>$ Cystic masses}

- In order of clock hours, first retroareolar, then 1 to 12

3 Please only mention BIRADS 3 and 4 cysts, those in region of relevant findings in other imagings, and those in region of clinician interest as requested in their order.

3 Multiple cystic lesions may be defined in a row.

$\checkmark$ In each line, please first write the location (.... O'clock), then if needed the zone (NZ, MZ, or FZ) and the depth (...mm from skin), then the features as needed (intracystic mass, septations ....).

\& Right breast:

- As above

- Axillary lymph nodes:

For normal or reactive lymph nodes please only mention nonsignificance, and do not mention size and other features

Please mention when lymph nodes are relevant to a clinical or other imaging finding

For suspicious nodes, please mention features as needed (cortical thickness, hilum changes, extracapsular invasion, matted nodes, etc.), the BIRADS of that specific lymph node and the recommendation (short-term follow-up, tissue diagnosis...)

- Other important findings (skin changes, duct changes, seroma, etc.)

- BIRADS

Recommendations (follow-up/further imaging/suggestion of tissue diagnosis, etc. for breast or axillary lesions)

Please do not mention type of surgical management

ID: Identification; ACR: American College of Radiology; BIRADS: Breast Imaging Reporting and Data System

in order to ascertain the site of the lesion and a correct comparison with the previous BUS.

After using the format, the survey showed a high level of agreement of the team members with ease of use, clarity, conciseness, believed that the organization of the report took more time. This could be permanently true or may be temporary due to the novelty of the structure, which might take time to get used to by the radiologists and their assistants who are preparing the report. Two of the surgeons thought the format was time-consuming, and one could not decide about the time; these were considering the time for preparing the 
Table 4. Survey questions

\begin{tabular}{lll} 
Number & Abbreviation* & Question \\
\hline 1 & Easy & The format is easy to use \\
3 & Clear & The definitions are clear \\
4 & Concise & The format is concise and useful \\
5 & Comprehensive & The format contains all key elements \\
6 & Sorted & The format contains necessary classifications \\
7 & Ordered & The arrangement is appropriate for comparison of two BUSRs \\
8 & Indispensable & The present details cannot be deleted \\
9 & Time-saving & Using this format takes less time \\
10 & Superior & The format increases the quality of the BUSR \\
$11 * *$ & Ongoing & I am eager to use the format in all my BUSRs
\end{tabular}

*Abbreviations demonstrating the subject of each question in calculations and in the figures, ** Question number 11 is not depicted in the figure because it had no direct relation with the format assessment.

BUSR: Breast ultrasound report

report; the two other surgeons agreed that in comparison with the BUS reports they were receiving before the study, this one took much less time to read, understand, and specially compare with the previous report. The indispensability of all details and whether the report could be further shortened were also questionable for some of the members.

Our study had some limitations. First and foremost, the COVID-19 situation disrupted the usual flow of patients and BUS. In addition, the users of the format were the same as the designers. Therefore, the study should also be performed by other users in other centers provide a more valid assessment of the proposed format.

In conclusion, we propose our format as a user-friendly format for BUS reports, which may be used and introduced as an adjunct to the BIRADS ultrasound lexicon. The format should be applied for a longer time in university hospitals in order to find out if the apparent time-consuming nature for radiologists would be solved by routine use. Also, the format should be tested in other centers in order to ascertain its positive features.

\section{Acknowledgements}

We would like to acknowledge Dr. Mehrnoush Hadadi (Dezfool, Iran), Dr. Mehdi Ghassemi (Andimeshk, Iran) and Mrs. Marzieh Orooji (Tehran, Iran) for their kind collaboration in providing and collecting BUS reports from various centers and cities.

Ethics Committee Approval: This project was supported by the ViceChancellor in Research Affairs of Tehran University University of Medical Sciences and was assessed and ethically approved by its Ethics Committee (Ethics code: IR.TUMS.VCR.REC.1 397.846).

Informed Consent: Informed consent was obtained.

Peer-review: Externally peer-reviewed.

\section{Authorship Contributions}

Conception: S.A., B.E., M.A., N.A., A.A., K.B., L.B., A.E., M.G., M.R., N.S., A.Y., R.O. Al.A.; Design: S.A., B.E., M.A., N.A., A.A., K.B., L.B., A.E., M.G.,
M.R., N.S., A.Y., R.O., Al.A.; Supervision: S.A., B.E., M.A., N.A., A.A., K.B., L.B., A.E., M.G., M.R., N.S., A.Y., R.O.; Materials: S.A., B.E., M.A., N.A., A.A., K.B., L.B., A.E., M.G., M.R., N.S., A.Y., R.O., Al.A.; Data Collection or Processing: S.A., B.E., M.A., N.A., A.A., K.B., L.B., A.E., M.G., M.R., N.S., A.Y., R.O., Al.A.; Analysis or Interpretation: S.A., B.E., M.A., N.A., A.A., K.B., L.B., A.E., M.G., M.R., N.S., A.Y., R.O., Al.A.; Literature Search: S.A., B.E., M.A., N.A., A.A., K.B., L.B., A.E., M.G., M.R., N.S., A.Y., R.O., Al.A.; Writing: S.A., B.E., M.A., N.A., A.A., K.B., L.B., A.E., M.G., M.R., N.S., A.Y., R.O., Al.A.

Conflict of Interest: No conflict of interest was declared by the authors.

Financial Disclosure: This study was supported by grant in aid of Tehran University of medical Sciences (no: \# 97-03-218-40362).

\section{References}

1. Maajani K, Jalali A, Alipour S, Khodadost M, Tohidinik HR, Yazdani $\mathrm{K}$. The global and regional survival rate of women with breast cancer: a systematic review and meta-analysis. Clin Breast Cancer 2019; 19: 165177. (PMID: 30952546) [CrossRef]

2. Bray F, Ferlay J, Soerjomataram I, Siegel RL, Torre LA, Jemal A. Global cancer statistics 2018: GLOBOCAN estimates of incidence and mortality worldwide for 36 cancers in 185 countries. CA Cancer J Clin 2018; 68: 394-424. (PMID: 30207593) [CrossRef]

3. Posso M, Louro J, Sánchez $M$, Román $M$, Vidal $C$, Sala $M$, et al. Mammographic breast density: how it affects performance indicators in screening programmes? Eur J Radiol. 2019;110: 81-87. (PMID: 30599878) [CrossRef]

4. Dawson C, Armstrong MW, Michaels J, Faber RG. Breast disease and the general surgeon. II. Effect of audit on the referral of patients with breast problems. Ann Royal Coll Surg Engl 1993; 75: 83. (PMID: 8476191) [CrossRef]

5. Mendelson E, Böhm-Vélez M, Berg W, et al. ACR BI-RADS ultrasound. In ACR BI-RADS atlas, breast imaging reporting and data system. 5th ed. 2013:1-173. Philadelphia: Clinical Research Center: 2013.

6. Burnside ES, Sickles EA, Bassett LW, Rubin DL, Lee CH, Ikeda DM, et al. The ACR BI-RADS ${ }^{\bullet}$ experience: learning from history. J Am Coll Radiol 2009; 6: 851-860. (PMID: 19945040) [CrossRef] 
7. Lynge E, Vejborg I, Andersen Z, von Euler-Chelpin M, Napolitano G. Mammographic density and screening sensitivity, breast cancer incidence and associated risk factors in danish breast cancer screening. J Clin Med 2019; 8: 2021. (PMID: 31752353) [CrossRef]

8. von Euler-Chelpin M, Lillholm M, Vejborg I, Nielsen M, Lynge E. Sensitivity of screening mammography by density and texture: a cohort study from a population-based screening program in Denmark. Breast Cancer Res 2019; 21: 111. (PMID: 31623646) [CrossRef]

9. Hollingsworth $\mathrm{AB}$. Redefining the sensitivity of screening mammography: a review. Am J Surg 2019; 218: 411-418. (PMID: 30739738) [CrossRef]

10. Berg WA, Zhang Z, Lehrer D, Jong RA, Pisano ED, Barr RG, et al. Detection of Breast Cancer with Addition of Annual Screening Ultrasound or a Single Screening MRI to Mammography in Women with Elevated Breast Cancer Risk. JAMA 2021; 307: 1394-1404. (PMID: 22474203) [CrossRef]

11. Gharekhanloo F, Torabian S, Kamrani S. Survey of the role of combined screening method with ultrasonography in the diagnosis of breast cancer. Avicenna J Clin Med 2011; 17: 57-60. [CrossRef]
12. The American College of Radiology: ACR practice parameter for the performance of whole-breast ultrasound for screening and staging. Last Accessed Date: 24.07.2020. Available from: https://www.acr.org/-/media/ ACR/Files/Practice-Parameters/USWholeBreast.pdf [CrossRef]

13. Abdullah N, Mesurolle B, El-Khoury M, Kao E. Breast imaging reporting and data system lexicon for US: interobserver agreement for assessment of breast masses. Radiology 2009; 252: 665-672. (PMID: 19567644) [CrossRef]

14. Lazarus E, Mainiero MB, Schepps B, Koelliker SL, Livingston LS. BIRADS lexicon for US and mammography: interobserver variability and positive predictive value. Radiology. 2006; 239: 385-391. (PMID: 16569780) [CrossRef]

15. Costantini M, Belli P, Lombardi R, Franceschini G, Mulè A, Bonomo L. Characterization of solid breast masses: use of the sonographic breast imaging reporting and data system lexicon. J Ultrasound Med 2006; 25 : 649-659. (PMID: 16632790) [CrossRef]

16. Levy L, Suissa M, Chiche JF, Teman G, Martin B. BIRADS ultrasonography. Eur J Radiol 2007; 61: 202-211. (PMID: 17215097) [CrossRef] 